\title{
Evaluation of spectral CT in differentiating metastatic from non-metastatic lymph nodes in non-small cell lung cancer(NSCLC)
}

\section{Funan Wang}

Xiamen Branch, Zhongshan Hospital, Fudan University

\section{Yanwei Wang}

Affiliated Xiamen No.2 Hospital of Xiamen Medical College

Gang Guo

Affiliated Xiamen No.2 Hospital of Xiamen Medical College

Liuhong Zhu ( $\square$ zhuliuhong@163.com)

Research article

Keywords: metastatic lymph nodes, non-small lung cancer, spectral CT

Posted Date: February 25th, 2020

DOI: https://doi.org/10.21203/rs.2.24477/v1

License: (c) (1) This work is licensed under a Creative Commons Attribution 4.0 International License. Read Full License 


\section{Abstract}

\section{Background}

Lung cancer is the main cause of tumor-correlated deaths, analysis of lymph nodes is crucial to staging of lung cancer. The purpose of the study is to explore the importance of spectral CT imaging in the difference prognostic of metastatic and non-metastatic mediastinal lymph nodes in non-small cell lung cancer.

\section{Methods}

A retrospective examination of 76 patients with non-small cell lung tumor who underwent spectral CT was performed. Quantifiable GSI (Gemstone spectral imaging) parameters (eg, $40 \mathrm{keV}$, iodine concentration, water concentration) were calculated in non-contrast, arterial and venous phase in 110 mediastinal lymph nodes using AW4.6 (GE HEALTHCARE, USA).

\section{Results}

The CT values of $40 \mathrm{kev}, \lambda \mathrm{HU}$ (The slope of Hounsfield unit curve) and IC(values of iodine concentration values), WC(values of water concentration) measured at the arterial or venous phase were not significantly different from those of metastatic growth lymph nodes $(P>0.05)$. The net value of Arterial phase (nIAP,net value of iodine concentration in Arterial phase) and vein phase (nIVP, net value of iodine concentration in vein phase) were calculated. The value of nIAP was the difference between IAP (iodine concentration of arterial phase) and INCP (iondine concentration of non-contrast phase), while the value of nIVP was the difference between IVP (iodine concentration of venous phase) and INCP. There stood no noteworthy difference in nIAP amid metastatic lymph nodes and non-metastatic lymph nodes $(P=$ 0.110). There was a substantial difference in nIVP amid metastatic lymph nodes and non-metastatic lymph nodes $(P=0.001)$.

\section{Conclusions}

Compared with qualitative assessment with conventional CT imaging features, quantitative GSI parameters (nIVP) showed higher accuracy for the preoperative diagnosis of mediastal lymph nodal metastases in patients with NSCLC.

\section{Background}

Lung cancer is the main cause of tumor-correlated deaths in China, according to 2016 cancer statistics. Non-small cell lung cancer is the greatest conjoint histological subtype of lung cancer, and its accurate diagnosis is very important (1). Although treatment has improved, the diagnosis of NSCLC growth remains poor, with a 5-year endurance rate of only $11-15 \%$ (2). Radical resection, including total tumor resection and total lymphadenectomy, is the standard treatment for my-IIIB stage NSCLC. Proper analysis is important for determining diagnosis and selecting appropriate treatment methods, especially for lymph 
nodes. Transfer is considered important. CT is the most widely used and most commonly used imaging modality for evaluating the mediastinum of lung cancer. However, the traditional CT prognostic of metastatic lymph swellings in lung malignancy is not accurate (3), and PET / CT also avoids the exposure of minor metastatic lymph swellings (4).

Radical resection, including total tumor resection and total lymph node dissection, is the typical cure for stage I-IIIB NSCLC and requires a non-invasive approach to describe mediastinal lymph nodes more accurately.

A dual-energy data set based fast kilovoltage switching spectral CT imaging mode is proposed, which can maintain the potential of reconstructing arbitrary monochromatic images in the range of 40-140 keV and quantify the decomposed image measurement (5).

However, as far as we know, the quantity of GSI in the prior operative prognostic of lymph node metastasis in patients with NSCLC growth has not been assessed. Less studies in these studies have examined the reproducibility of their results.

The purpose of the study was to assess the value of spectral CT in differential analysis of mediastinal metastatic lymph nodes and non-metastatic lymph nodes in NSCLC.

\section{Methods}

\subsection{Patients}

Our institutional ethics committee has approved a prospective study, and informed consent has been signed by all patients. From October 2016 to September 2017, 84 patients with confirmed primary lung cancer performed non-enhanced and dual-phase enhance thoracic CT scans. All patients who experienced surgery inside 7 days afterward the CT scan and were eligible for the subsequent measures were qualified for addition in the research.

Inclusive criteria: (1) All patients did not receive preoperative treatment (radiotherapy and / or chemotherapy); (2) axial images of lymph nodes with diameter $>5 \mathrm{~mm}$ at the short-axis views; (3) Benign and metastatic lymph nodes were proved by pathology. (4) all patients were given out by histopathology as a non-small cell lung cancer.

Eight cases were excluded after CT scanning because of their refusal of surgery $(n=4)$, or lack of specific pathological results $(n=4)$.

Figure 1 shows the detailed timeline of the research protocol. The case accumulation process was conducted by two radiologists, with more than 5 years of thoracic imaging experience.

\subsection{GSI Quantitative Parameters Acquisition}


The high-definition CT system (Disco. CT750 HD, GE Medical Group, Milwaukee, Wisconsin, USA) was used to perform dual-phase enhanced chest examinations using a chest GSI model. Then a 20GA needle was injected through the anterior cubital vein at a rate of $3 \mathrm{ml} / \mathrm{sec}$ by means of a $70-120 \mathrm{ml}(1.5 \mathrm{ml} / \mathrm{kg})$ nonionic iodine distinction agent $(320 \mathrm{mg} / \mathrm{ml}$, loversol, China). The first non-contrast phase scan was performed prior to iodine injection, and then the optimal arterial phase (AP) was obtained using the Intelligent Preparation Protocol (triggered by $120 \mathrm{Hu}$ of the thoracic aorta), then the venous phase (VP) was acquired in 55 s delay. Other GSI parameters wereic as follows: tube revolution time, 0.6 seconds; pitch, 1.375; tube current, $260 \mathrm{~mA}$; volume CT dose index, $7.64 \mathrm{mGy}$, at each stage of the chest GSI model. The reconstructed images, slice thickness and interval of GSI data were all $1.25 \mathrm{~mm}$, and the regions of interest around each marker were drawn on the reconstructed $70 \mathrm{keV}$ monochromatic vein images. The area of interest ought to be as great as possible, including the whole lymph node, and carefully remove the surrounding fat necrosis and calcification.

\subsection{Quantitative analysis of LNs(lymph nodes)}

Qualitative analysis of $70 \mathrm{keV}$ monochromatic images was performed at GE Advantage workstation 4.6 (GE Medical Group, USA) by two radiologists with masked pathology ( 5 and 9 years of experience, respectively). Any differences were resolved through consensus. All extents were performed on two successive sections for two times, and the average values were deliberated.

The extent of the goal lymph node was calculated at the long-axial view through the energy level of $70 \mathrm{kV}$ in GSI mode. Because the conventional $120 \mathrm{kVp}$ CT scan has the average energy of 70-kev. AP and VP were selected from the average CT value of nodes in $40 \mathrm{keV}$ images, because materials could be more easily distinguished from low keV images by adaptive iterative satisfaction improvement (ASIR). In the image of material decomposition, GSI observers measured the density of matter, and the average iodine concentration (IC, $\mathrm{mg} / \mathrm{ml}$ ) and water concentration $(\mathrm{WC}, \mathrm{mg} / \mathrm{ml})$ were measured with iodine and waterbased materials. According to the total body weight and the total amount of non-ionic iodine contrast agents, a similar personalized test was conducted for each patient. In addition, our aim was to find a convenient clinical application, so iodine concentrations were not standardized. The GSI Viewer software spontaneously calculates the CT quantity of each energy level as of the iodine-based material decomposition (MD) appearance at the virtual monochromatic image level (40-100KV). The slope of the calculated spectral curve $(\lambda \mathrm{hu})$ is calculated according to this formula, such as the difference between the secondary CT values (40 and $100 \mathrm{keV}$ ) divide by the energy variance (60 keV) from the Hounsfiel units curve, $\lambda(\mathrm{Hu})=($ CT40 kev-CT $100 \mathrm{kev}) /(100-40)$.

\subsection{One to one comparison criteria for LNs}

LNs is used to promote one to one comparison of radiological pathology of LN. Regional lymph nodes are the seventh edition of the International Association for Lung Cancer Research (IASLC) revised according to staging criteria. Surgical resection of lymph nodes (such as location, size, record of adjacent structures), marking, and sending pathological examinations are described in detail. If all the progression occurs in each group of lymph node metastases, it is determined that the lymph node CT images are 
taken as being metastatic lymph nodes in each set. The similar method was applies to non-metastatic LNs. When the collected LNs includes metastatic and benign lymph nodes, we attempt to trail the size and distance of the regional LN based on the tumor (6) .

\subsection{Statistical analysis}

Statistical investigation using SPSS 19 edition data (SPSS, Chicago, IL, the United States), P $<0.05$ was considered to be statistically significant. The quantitative variable is expressed by mean \pm standard deviation (SD). The differences between AP and VP, nIAP( net value of iodine concentration in Arterial

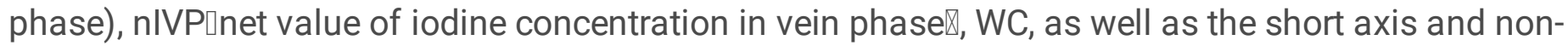
metastatic lymph nodes in IC were related by t-test of separated samples. Receptor operating characteristic (ROC) curve generation helps to establish a threshold amid the parameters of metastatic and non-metastatic lymph nodes. The prognostic ability is known by calculating the area underneath the $\mathrm{ROC}$ arc. The optimal threshold is determined based on the highest $\mathrm{J}$ statistic. When the best threshold is used, the sensitivity and specificity of these quantitative parameters are calculated. The $P$ value was less than 0.05 , and the difference was statistically substantial.

\section{Results}

\subsection{Lymph nodes and patients}

Among the 104 cases of lung cancer, there were 49 circumstances of adenocarcinoma, 38 circumstances of squamous cell carcinoma and 17 cases of atypical carcinoma. 20 patients without enhanced examination was excluded. 181 lymph nodes in 76 patients were labeled on preoperative CT images. A total of 71(39.2\%) lymph nodes in patients with NSCLC were dissected, and 110 (60.7\%) of these nodes, including 57 metastatic and 53 benign lymph nodes, were successfully matched and labeled during surgery. Table 1 showed the distribution of all 110 lymph nodes. 
Distribution of All 110 Lymph Nodes.

\begin{tabular}{|lll|}
\hline Levels & Benign Lymph Nodes $(\mathbf{n}=\mathbf{5 3})$ & Metastatic Lymph Nodes $(\mathbf{n}=\mathbf{5 7})$ \\
\hline 1 & 0 & 0 \\
2 & 2 & 2 \\
3 & 3 & 5 \\
4 & 17 & 16 \\
5 & 11 & 13 \\
6 & 5 & 7 \\
7 & 15 & 14 \\
8 & 0 & 0 \\
\hline 9 & 0 & 0 \\
\hline
\end{tabular}

\subsection{Quantitative parameters' inquiry of metastatic and non- metastatic lymph nodes}

\subsubsection{Size of LNs}

The average short-axis diameter of all metastatic lymph nodes $(10.81 \pm 2.44 \mathrm{~mm}$, range: $6.9-16.1 \mathrm{~mm})$ was meaningfully higher than that of non-metastatic lymph lesions $(10.03 \pm 2.05 \mathrm{~mm}$, range: $6.5-$ $14.5 \mathrm{~mm})(P=0.019))$ and the $9.35 \mathrm{~mm}$ threshold would produce $59 \%$ sensitivity, $68 \%$ specificity, and $65.4 \%$ accuracy.. According to the established criteria, only $60(67 / 110)$ lymph nodes $(>10 \mathrm{~mm}), 49$ metastatic lymph nodes $(>10 \mathrm{~mm}(44)$, and $18<10 \mathrm{~mm}(16 \%)$ were found. (Table 2$)$.

Table 2

Difference of CT morphologic diagnosis between lymph node metastasis and pathologic diagnosis.

\begin{tabular}{|llll|}
\hline Pathological confirmed Groups & \multicolumn{2}{c|}{ Conventional CT Features } & Total \\
\cline { 2 - 3 } & $\geq 10 \mathrm{~mm}$ & $<10 \mathrm{~mm}$ & \\
\hline Benign Lymph Nodes & 18 & 35 & 53 \\
\hline Metastatic Lymph Nodes & 49 & 8 & 57 \\
\hline Total & 67 & 43 & 110 \\
\hline
\end{tabular}


Table 3

Comparison GSI Quantitative Parameters of Benign with Metastatic Lymph Nodes.

\begin{tabular}{|llll|}
\hline GSI Parameters & Benign Lymph Nodes & Metastatic Lymph Nodes & P Value \\
\hline Short Diameter & $10.03 \pm 2.06$ & $10.81 \pm 2.44$ & 0.019 \\
\hline NC CT value & $43.15+18.79$ & $56.43+10.96$ & 0.153 \\
\hline NC $\lambda$ HU & $2.49+1.76$ & $4.15+3.34$ & 0.227 \\
\hline NC IC & $2.07 \pm 1.44$ & $3.27 \pm 2.79$ & 0.111 \\
\hline NC WC & $1025.84+11.65$ & $1027.5+9.88$ & 0.857 \\
\hline AP CT value & $120.77+67.14$ & $120.96+76.70$ & 0.075 \\
\hline AP $\lambda$ HU & $1.17+0.77$ & $1.09+0.87$ & 0.425 \\
\hline AP IC & $10.43+8.10$ & $10.72+9.01$ & 0.407 \\
\hline AP WC & $1030.54+15.07$ & $1040.43+14.89$ & 0.106 \\
\hline VP CT value & $131.39+45.61$ & $108.50+41.67$ & 0.057 \\
\hline VP $\lambda$ HU & $1.48+0.60$ & $1.71+0.58$ & 0.057 \\
\hline VP IC & $12.52+5.13$ & $9.86+4.86$ & 0.059 \\
\hline VP WC & $1036.06+11.60$ & $1040.43+14.89$ & 0.699 \\
\hline $\begin{array}{l}\text { NC: Non-contrast; AP: Arterial phase; VP: Venous phase; } \lambda \text { HU: The slope of Hounsfield unit curve; IC: } \\
\text { values of iodine concentration values; } \text { WC: values of water concentration; data are average values } \pm \\
\text { standard deviations; } P<0.05 \text { demonstrates a statistically marked difference. }\end{array}$ \\
\hline
\end{tabular}

\subsubsection{CT value of lymph nodes at $40 \mathrm{kev}, \lambda_{\mathrm{HU}}, \mathrm{IC}$, and WC}

The CT values of $40 \mathrm{kev}, \lambda_{\mathrm{HU}}$, IC and WC in arterial and venous metastatic lymph nodes and nonmetastatic lymph nodes stood not significantly different from those in benign lymph nodes $(P>0.05)$. (figures) 2 and 3).

The value of 3.2.3.nIAP and nIVP lymph nodes

The net value of IC in AP (nIAP) and VP (nIVP) was calculated according to the formula nIAP = IAP(iodine concentration in Arterial phase )-INCP (iodine concentration in non-contrast period) and nIVP = IVP(iodine concentration in vein phase)-INCP. There was no substantial variance amid nIAP metastasis and non metastatic lymph nodes $(P=0.110)$. Fortunately, there was a substantial change in nIVP amid metastatic and non-metastatic lymph nodes $(P=0.001)$ (Table 4). 
Table 4

Comparison GSI Quantitative Parameters of benign with Metastasis Lymph Nodes .

\begin{tabular}{|llcc|}
\hline GSI Parameter & Benign Lymph Nodes & Metastatic Lymph Nodes & P Value \\
\hline nIAP & $8.36+7.79$ & $5.87+12.10$ & 0.11 \\
\hline nIVP & $10.453 \pm 4.68$ & $6.39 \pm 4.19$ & 0.001 \\
\hline $\begin{array}{l}\text { nIAP: net value of iodine concentration in Arterial phase; nIVP: net value of iodine concentration in } \\
\text { Venous phase }\end{array}$ & & \\
\hline
\end{tabular}

\subsubsection{ROC analysis}

The ROC arc nIVP of lymph node extent was determined in VP and the optimum verge for each measureable parameter was analyzed (Fig. 3). All ROC arches were higher than baseline. The area under the ROC curve of NIVP (AUC) $=0.743$ is larger than that of the short axis (AUC $=0.654$ ). When the ideal threshold of NIVP was 8.185 , the sympathy, specificity and total precision of lymph node metastasis were $67.0 \%, 76 \%, 0 \%$ and $74.3 \%$, respectively.

\section{Discussion}

This research evaluated the variance in iodine content and short-axial view amid non-metastatic and metastatic mediastinal lymph swellings. CT is the most commonly used and most commonly used imaging examination method for mediastinum of lung cancer. Lymph node metastasis and extra nodal expansion are widely used to identify the size, extent and enhancement of lymph nodes. However, conventional CT uses a single-energy X-ray ray to capture images, wherever pixel values and Housfield value are based on the number of photons reaching the detector and the number of photons absorbed by different tissues. (that is, calcified plaque and iodine comprising blood), and the prospect of distinguishing different constituents in the structure is limited. The sensitivity and specificity of conventional CT in diagnosing metastatic lymph nodes recorded $51 \%$ and $85 \%$ correspondingly. Of course. The incidence of NSCLC is relatively low, and its comparative value is also limited.

The gemstone spectral CT imaging (GSI) mode instantly collects a rotating data between $80 \mathrm{kVp}$ and 140 kVp. Compared with traditional multi-row CT, spectral CT not only provides 40-140 keV virtual monochromatic spectral images, consequently provides material decay images, effective atomic quantity images and spectral curves, thus magnifying the small differences between diverse tissue sources (7-9). The measurable parameters of spectral CT can be applied in the clinical identification of pulmonary embolism and in the disparity analysis of benign and malignant growths.

lodine levels in metastatic and non-metastatic LNs are mainly evaluated by averages. As we all know, LNs is not an isomorphic organizational structure. In fact, LNs is collected along the cortex of the oocyst organ, mainly B lymphocytes in the follicular space and several T lymphocytes (in the follicular space), 
and the medulla is transmitted to the lymphocytes and blood vessels of the afferent and transitive organs $(10,11)$. Inoculation and growth of tumor cells in LNs induce neovascularization, so higher iodine levels can be anticipated in metastatic lymph nodes $(12,13)$.

After intravenous injection, the contrast medium first enters the blood system. Therefore, intravascular imaging leads to arterial enhancement. Then, intravascular contrast gradually spread to extravascular tissue, so the enhancement of venous tissue is caused by intravascular and interstitial space (14).

Post-contrast evaluation is one of the oldest methods for evaluating malignant process function and an important component of CT or MRI evaluation, but it is used for staging and follow-up (15). The enhancement of contrast after attenuation of any tissue reflects the level of angiogenesis, which may be affected by the angiogenesis of malignant structures. But the absolute attenuation value is not only related to iodine concentration, but also related to the pathological process of malignant lesions such as necrosis and hemorrhage.

It also depends on the observer, such as the precise location of ROI(region of interesting) in the lesion (16).

Previous studies have confirmed the reliability and accuracy of the experiment, so spectral data set analysis is a very useful and simple, semi-enhanced $(17,18)$ automatic quantitative technique.

In this study, we confirmed the decomposition of iodine water, indicating a significant difference between LNs and lymph node metastasis $(P<0.05)$.

In our study, we confirmed that the nIVP value of metastatic lymph nodes was significantly lower than that of iodine-based substance decomposition images without lymph node metastasis. This difference is mainly in lymph node metastasis and lymphatic damage in the number of arteriovenous malformations increased, resulting in rapid increase in customs clearance.

We know that lymph node CT depends not only on the concentration of intra-atrial and intra-atrial contrast media, but also on early arterial phase enhancement, as well as extravascular / outdoor, which is the reason for late (venous) enhancement and metastatic infiltration. This is an important reason for lymph node evaluation after multi CT circulatory examination. In addition, the relationship between attenuation of lung cancer and FDG uptake is unclear because of the volume perfusion parameter (19).

With angiogenesis, the blood supply of metastatic lymph nodes is different from that of inflammatory lymph nodes. The mean peak attenuation value of dynamic CT reflects microvessel density of lung adenocarcinoma (20). Metastatic tumors (including metastatic lymph nodes) are associated with the pathological and pathophysiological characteristics of primary malignant tumors.

It is well clear that the pathophysiological variations affecting tissue blood bulk or vascular penetrability will be replicated for enrichment (21). Prior histopathological studies showed non metastatic LN had a 
greater vascular sum than metastatic LN. The authors speculate that the rich vascular distribution of natural LN eliminates the need to develop additional blood vessels to grow metastatic cells (22).

In terms of radiation dosage, the total dose-length product (DLP) of the two-phase enhanced scans was $(548.3+90.7) \mathrm{mGy} \mathrm{cm}$. This is the same as the traditional 64 level CT dual phase scanning.

This study has certain limitations and necessity. First, there is no standardized contrast in the aorta, which improves the contrast in all cases, but the total contrast volume is injected according to individual weight. Our main objective is to find a simple clinical application. It is. Second, despite our efforts to make a one-to-one matching of LN radiopathology, we were unable to make a perfect match. Lymph nodes were excluded from the study below $5 \mathrm{~mm}$, because the measurement uncertainty was attributable to incomplete matching. 4. Although the iodine content represented by iodine water decomposition was significantly different amid normal and metastatic lymph nodes, the quantity of lymph nodes $(n=110)$ in this investigation was very little to produce a cut-off value to differentiate the two. There is considerable overlay in classroom assessment. No other method (PET or CT perfusion) was used for comparison. Dissimilar histological types of non-small cell lung cancer at different stages were studied.It was assumed that all lymph nodes are independent, although some lymph nodes belong to the same patient.

\section{Conclusion}

Compared with volume perfusion CT, quantitative iodine uptake of GSI is an effective method for evaluating mediastinal lymph nodes with lower radiation. Quantitative GSI parameters, especially nIVP, are more accurate in preoperative diagnosis of NSCLC metastasis lymph nodes than conventional CT qualitative evaluation. The value of nIVP as a intratumoral contrast enhancement index can help predict lymph node metastasis and invasion. It is necessary to further verify the contribution of iodine related parameter evaluation.

\section{Abbreviations}

NSCLC: non-small cell lung cancer; LNs: lymph nodes; GSI: Gemstone spectral imaging; NC: Non-contrast; AP: Arterial phase; VP: Venous phase; $\lambda \mathrm{HU}$ : The slope of Hounsfield unit curve; IC: values of iodine concentration values; WC: values of water concentration; IAP :iodine concentration of arterial phase; IVP :iodine concentration of vein phase; INCP: iondine concentration of non-contrast phase; nIAP: net value of iodine concentration in Arterial phase; nIVP: net value of iodine concentration in Venous phase.

\section{Declarations}

Ethics approval and consent to participate: The study was Approvel by Fudan University Zhongshan Hospital Xiamen Branch ethics committee.

Consent for publication: Written informed consent was obtained from the patients. 
Availability of data and materials: The datasets used and/or analysed during the current study are available from the corresponding author on reasonable request.

Competing interests: The authors declare that they have no competing interests.

Funding: Not applicable

Authors' contributions: Liuhong zhu analyzed and interpreted the patient data regarding lung cancer patients. Funan Wang,who major contributor in writing the manuscript, performed the spectral CT examination with Yanwei Wang. Qihua cheng was responsible for revision. All authors read and approved the final manuscript

Acknowledgements: Not applicable

\section{References}

1. Chen W, Zheng R, Baade PD, Zhang S, Zeng H, Bray F, et al. Cancer statistics in China, 2015. CA Cancer J Clin. 2016;66(2):115-32.

2. Raungrut P, Wongkotsila A, Lirdprapamongkol K, Svasti J, Geater SL, Phukaoloun M, et al. Prognostic significance of 14-3-3gamma overexpression in advanced non-small cell lung cancer. Asian Pac $\mathrm{J}$ Cancer Prev. 2014;15(8):3513-8.

3. Silvestri GA, Gould MK, Margolis ML, Tanoue LT, McCrory D, Toloza E, et al. Noninvasive staging of non-small cell lung cancer: ACCP evidenced-based clinical practice guidelines (2nd edition). Chest. 2007;132(3 Suppl):178S-201S.

4. Silvestri GA, Gonzalez AV, Jantz MA, Margolis ML, Gould MK, Tanoue LT, et al. Methods for staging non-small cell lung cancer: Diagnosis and management of lung cancer, 3rd ed: American College of Chest Physicians evidence-based clinical practice guidelines. Chest. 2013;143(5 Suppl):e211S-e50S.

5. Lv P, Lin XZ, Li J, Li W, Chen K. Differentiation of small hepatic hemangioma from small hepatocellular carcinoma: recently introduced spectral CT method. Radiology. 2011;259(3):720-9.

6. Cho EY, Kim SH, Yoon JH, Lee Y, Lim YJ, Kim SJ, et al. Apparent diffusion coefficient for discriminating metastatic from non-metastatic lymph nodes in primary rectal cancer. Eur $\mathrm{J}$ Radiol. 2013;82(11):e662-8.

7. Yeh BM, Shepherd JA, Wang ZJ, Teh HS, Hartman RP, Prevrhal S. Dual-energy and low-kVp CT in the abdomen. AJR Am J Roentgenol. 2009;193(1):47-54.

8. Fuchs TA, Stehli J, Fiechter M, Dougoud S, Gebhard C, Ghadri JR, et al. First experience with monochromatic coronary computed tomography angiography from a 64-slice CT scanner with Gemstone Spectral Imaging (GSI). J Cardiovasc Comput Tomogr. 2013;7(1):25-31.

9. Geyer LL, Scherr M, Korner M, Wirth S, Deak P, Reiser MF, et al. Imaging of acute pulmonary embolism using a dual energy CT system with rapid kVp switching: initial results. Eur J Radiol. 2012;81(12):3711-8. 
10. Elmore SA. Histopathology of the lymph nodes. Toxicol Pathol. 2006;34(5):425-54.

11. Raudenbush SW BA. Hierarchical linear models: applications and data analysis methods, 2nd edn. Sage, London. 2002.

12. Nathanson SD. Insights into the mechanisms of lymph node metastasis. Cancer. 2003;98(2):413-23.

13. Pastushenko I, Van den Eynden GG, Vicente-Arregui S, Prieto-Torres L, Alvarez-Alegret R, Querol I, et al. Increased Angiogenesis and Lymphangiogenesis in Metastatic Sentinel Lymph Nodes Is Associated With Nonsentinel Lymph Node Involvement and Distant Metastasis in Patients With Melanoma. Am J Dermatopathol. 2016;38(5):338-46.

14. Chin A Yi KSL, Byung-Tae Kim,et al. Efficacy of helical dynamic CT versus integrated PET/CT for detection of mediastinal normal metastasis in non-small cell lung cancer. Am J Roentgenol. 2007;188:318-25.

15. Tawfik AM, Razek AA, Kerl JM, Nour-Eldin NE, Bauer R, Vogl TJ. Comparison of dual-energy CTderived iodine content and iodine overlay of normal, inflammatory and metastatic squamous cell carcinoma cervical lymph nodes. Eur Radiol. 2014;24(3):574-80.

16. Kim YN, Lee HY, Lee KS, Seo JB, Chung MJ, Ahn MJ, et al. Dual-energy CT in patients treated with anti-angiogenic agents for non-small cell lung cancer: new method of monitoring tumor response? Korean J Radiol. 2012;13(6):702-10.

17. Godoy MC ND, Marchioni D et al. Basic principles and post processing techniques of dual-energy CT: illustrated by selected congenital abnormalities of the thorax. J Thorac Imaging. 2009;24:152-9.

18. Toepker M, Moritz T, Krauss B, Weber M, Euller G, Mang T, et al. Virtual non-contrast in secondgeneration, dual-energy computed tomography: reliability of attenuation values. Eur J Radiol. 2012;81(3):e398-405.

19. Miles KA, Griffiths MR, Keith CJ. Blood flow-metabolic relationships are dependent on tumour size in non-small cell lung cancer: a study using quantitative contrast-enhanced computer tomography and positron emission tomography. Eur J Nucl Med Mol Imaging. 2006;33(1):22-8.

20. Tateishi U, Nishihara H, Watanabe S, Morikawa T, Abe K, Miyasaka K. Tumor angiogenesis and dynamic CT in lung adenocarcinoma: radiologic-pathologic correlation. J Comput Assist Tomogr. 2001;25(1):23-7.

21. Miles KA. Tumour angiogenesis and its relation to contrast enhancement on computed tomography: a review. Eur J Radiol. 1999;30(3):198-205.

22. Naresh KN, Nerurkar AY, Borges AM. Angiogenesis is redundant for tumour growth in lymph node metastases. Histopathology. 2001;38(5):466-70.

\section{Figures}




\section{4 patients with lung cancer}

84 patients with

CT contrastenhance
$\mathrm{N}=20$ without CT contrastenhance

$\mathrm{N}=57$ positive

$\mathrm{N}=110$ lymph nodes(76 patients)
8 patients were excluded

\section{Figure 1}

Flow chart for lymph node examination and data handling in this research 


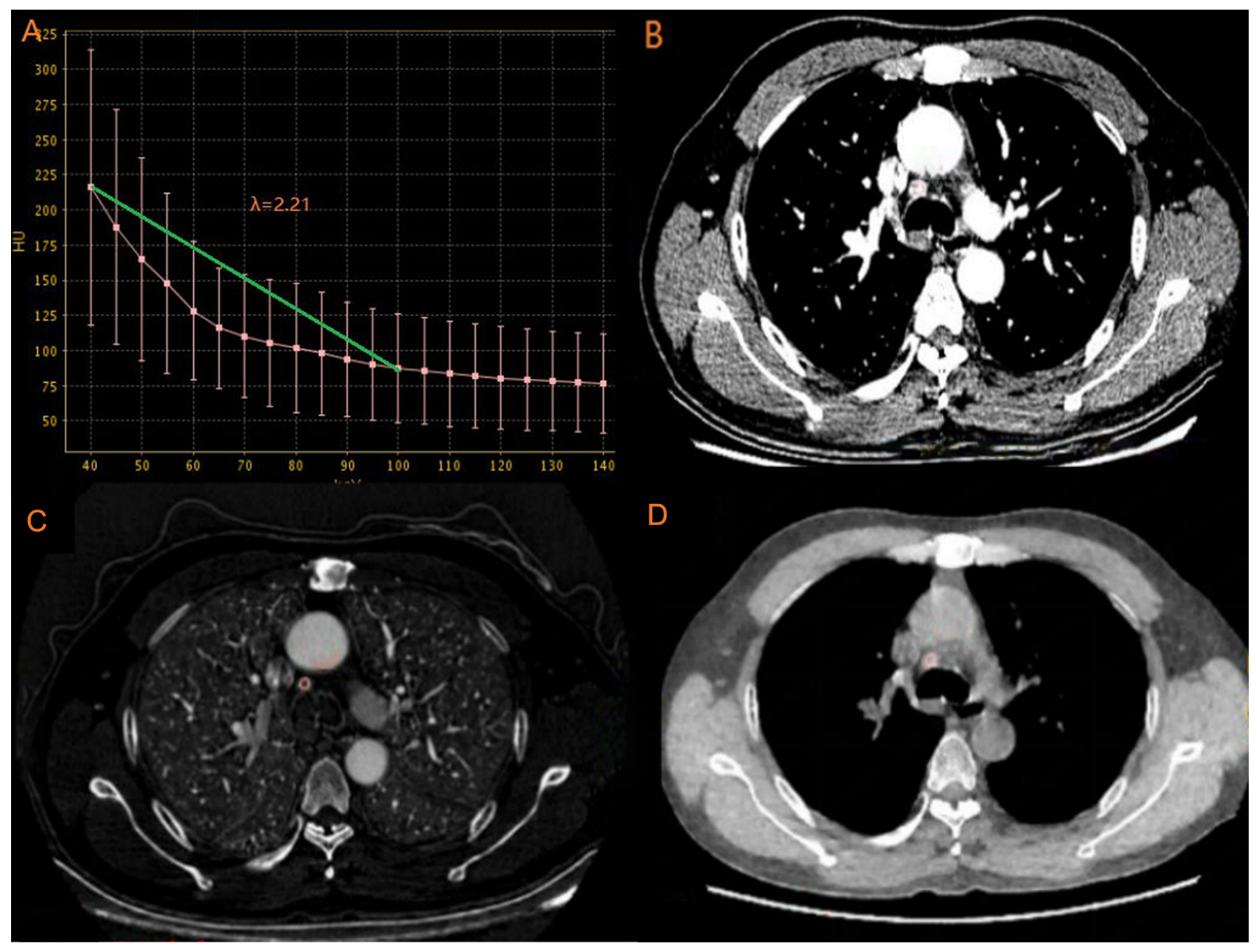

Figure 2

The patient diagnosed with NSCLC was 54 years old and histopathologically diagnosed. The Hursfield unit curve (lambda $\mathrm{Hu}$ ) of the fourth groups of lymph lumps during A: arterial phase was 2.21 . The 40 $\mathrm{keV}$ monochromatic images of BE:AP showed no expansion of the fourth lymph nodes. The diameter of the short axis is $6.1 \mathrm{~mm}$ and the CT value is $122.3 \mathrm{HU}$. The iodine concentration (IC) of C:AP iodine imaging was $8.41 \mathrm{mg} / \mathrm{C} 3$, and that of D:water-based material decomposition imaging was $1031.2 \mathrm{mg} / \mathrm{c} 3$. 


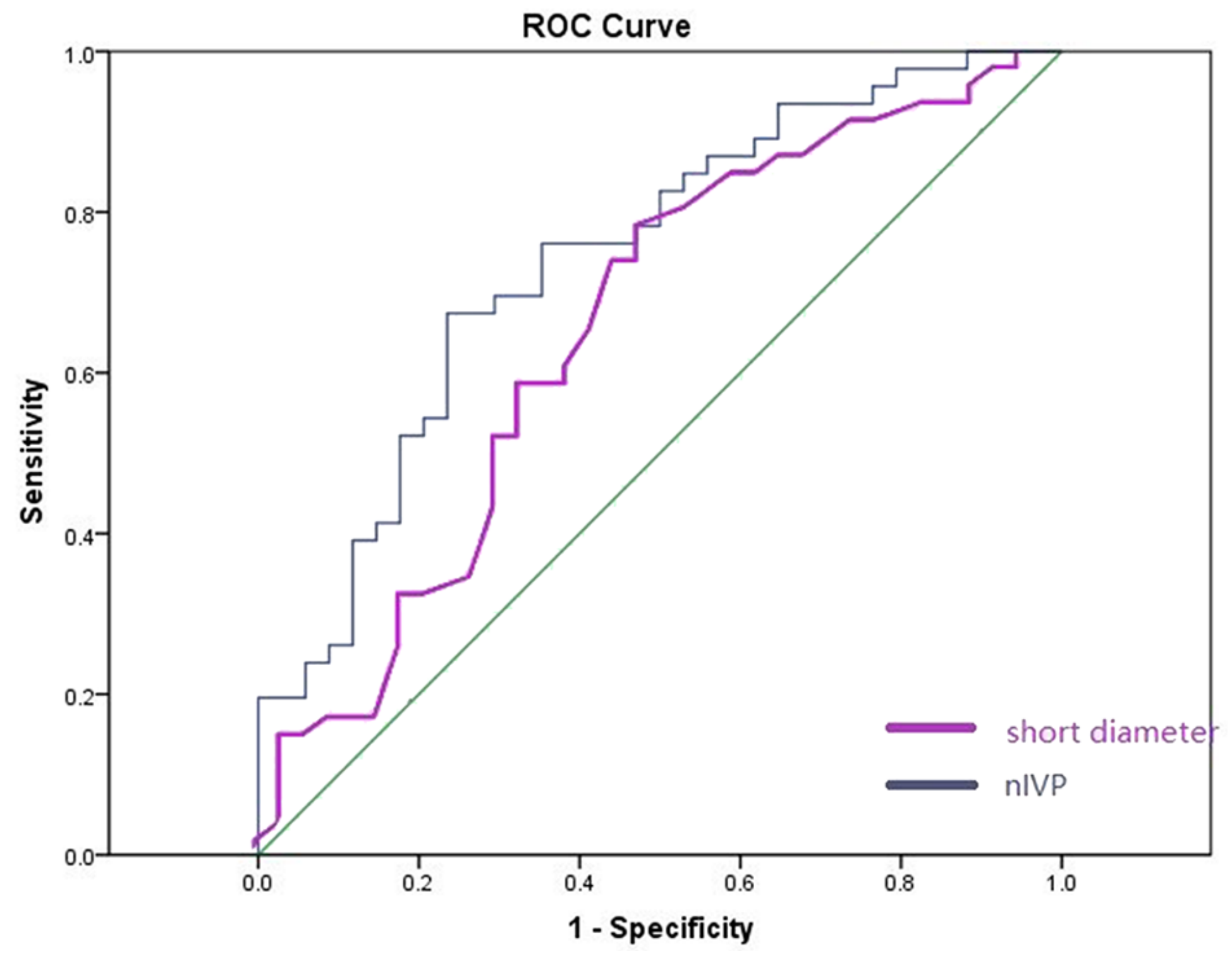

Figure 3

Graphs show receiver operating characteristic curves of nIVP and short diameter. The AUC of short diameter is 0.654 , and of nIVP is 0.743 . AUC $=$ area under the curve. 